\title{
Formation of a massive protostar through disk accretion
}

\section{SINFONI integral field spectroscopy of the M 17 silhouette disk and discovery of the associated $\mathrm{H}_{2}$ jet ${ }^{\star}$}

\author{
D. E. A. Nürnberger ${ }^{1}$, R. Chini ${ }^{2}$, F. Eisenhauer ${ }^{3}$, M. Kissler-Patig ${ }^{4}$, A. Modigliani ${ }^{4}$, R. Siebenmorgen ${ }^{4}$, \\ M. F. Sterzik ${ }^{1}$, and T. Szeifert ${ }^{1}$ \\ ${ }^{1}$ European Southern Observatory, Casilla 19001, Santiago 19, Chile \\ e-mail: dnuernbe@eso.org \\ 2 Astronomisches Institut der Ruhr-Universität Bochum, Universitätsstrasse 150, 44780 Bochum, Germany \\ 3 Max-Planck-Institut für extraterrestrische Physik, Giessenbachstrasse, 85748 Garching, Germany \\ ${ }^{4}$ European Southern Observatory, Karl-Schwarzschild-Strasse 2, 85748 Garching, Germany
}

Received 19 May 2006 / Accepted 12 December 2006

\begin{abstract}
Context. Recent observational results suggest that high-mass (proto-)stars form in a similar way to low- and intermediate-mass stars, i.e., via disk accretion of gas and dust.

Aims. To further characterize and understand the on-going physical processes associated with a large circumstellar disk, seen as a dark silhouette against the bright background of the M 17 H II region, we report and discuss new high angular resolution integral field spectroscopy performed in the $K$-band.

Methods. The data were taken with the Adaptive Optics (AO) supported near-infrared integral field spectrograph SINFONI at ESO's Very Large Telescope Yepun (VLT UT 4) as part of the science verification of this new instrument.

Results. Based on obtained $\mathrm{H}_{2} v=1-0 S(1)$ and $\mathrm{H}_{2} v=1-0 S(3)$ emission maps, we report the discovery of a $\mathrm{H}_{2}$ jet, which apparently arises from the suspected protostellar source(s) located at the very center of the disk. In addition, both diameter (about $4000 \mathrm{AU})$ and sub-structures of the innermost, densest part of the flared disk are infered from $\operatorname{Br} \gamma, \operatorname{Br} \delta$, and $\mathrm{He} I$ maps.

Conclusions. Because ejection of material through a jet/outflow is always linked to accretion of gas and dust either onto the circumstellar disk or onto the central (protostellar) source(s), the presence of a collimated $\mathrm{H}_{2}$ jet provides indirect but unquestionable evidence for ongoing accretion processes in the case of the M 17 disk. The high mass outflow and accretion rates of $>10^{-5} \mathcal{M}_{\odot} \mathrm{yr}^{-1}$ derived from the SINFONI data suggest that a star of rather high mass is forming.
\end{abstract}

Key words. stars: early-type - stars: formation - stars: pre-main sequence - stars: individual: M 17 silhouette disk ISM: individual objects: M 17

\section{Introduction}

Some recent observational results suggest that high-mass stars indeed form in a way similar to low- and intermediate-mass stars, i.e., via disk accretion processes. For example, high angular resolution dust continuum observations at millimeter wavelengths with the IRAM Plateau de Bure Interferometer (PdBI) reveal for the extremely young massive star-forming region IRAS $19410+2336$, a protocluster mass distribution that is consistent with the stellar initial mass function (Beuther \& Schilke 2004). Therefore, the initial mass function and the final masses of stars are apparently already determined at the time when the initial molecular cloud cores start to fragment. In addition, high angular resolution infrared (IR) imaging of the Galactic H II regions NGC 3603 and M 17 led to the discovery of high-mass protostellar candidates at the interface between H II region and

* Based on observations performed at the European Southern Observatory Very Large Telescope on Cerro Paranal, Chile as part of the SINFONI science verification, program ID 60.A-9041(A). Obtained data sets are publicly available via the ESO archive http://archive.eso.org/eso/eso_archive_main.html adjacent molecular cloud (Nürnberger 2003, 2004; Chini et al. 2004; Nürnberger et al. 2005). These observations take advantage of the presence of a cluster of early-type main-sequence stars, which provide a huge amount of energetic photons and powerful stellar winds capable of evaporating and dispersing the surrounding interstellar medium. This way, nearby young stars - which otherwise would be deeply embedded in their natal molecular cloud cores - are set free at a relatively early evolutionary stage and become detectable at near- and mid-IR wavelengths.

Here we want to focus on the M 17 region. We have discovered and characterized a tremendous opaque silhouette against the diffuse homogeneous background emission of the M 17 H II region (Chini et al. 2004; see Fig. 1, left panel). This silhouette, unambiguously seen in deep near-IR data taken with ISAAC and NAOS-CONICA at ESO's Very Large Telescope (VLT), is associated with an hourglass shaped nebula and surrounded by a large disrupting envelope. From ${ }^{12} \mathrm{CO}(1-0),{ }^{13} \mathrm{CO}(1-0)$, and $\mathrm{C}^{18} \mathrm{O}(1-0)$ data and the adjacent $3 \mathrm{~mm}$ continuum data obtained with PdBI, we estimate that the entire disk + envelope system contains a gas + dust mass of up to $110 \mathcal{M}_{\odot}$. Although 

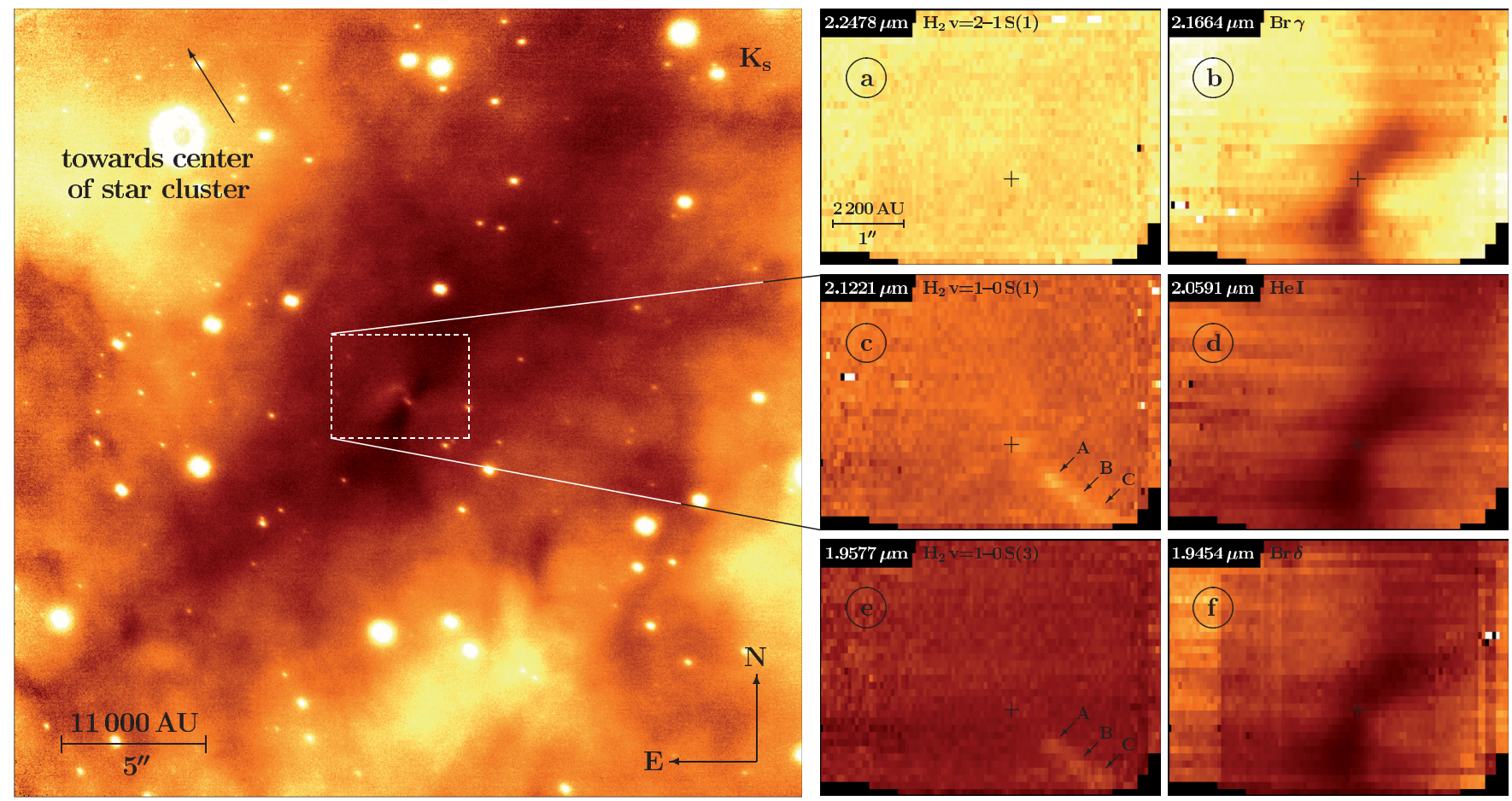

Fig. 1. Left: VLT + NACO $K_{\mathrm{s}}$-band image of the M 17 silhouette disk, recently discovered by Chini et al. (2004). The field of view is about $27^{\prime \prime} \times 27^{\prime \prime}$, which roughly corresponds to $60000 \mathrm{AU} \times 60000 \mathrm{AU}$ at the adopted M 17 distance of $2.2 \mathrm{kpc}$. The dashed box outlines the area of the VLT + SINFONI study. The bright artifact visible close to the upper left corner is caused by instrument internal reflexions of the AO reference star SAO 161357, which is located towards the East just outside of the displayed field of view. Right, panels a)-f): selected line maps extracted from the SINFONI data cube, each comprising a field of view of 4". $8 \times 3$ 3.' 6 , i.e., $10560 \mathrm{AU} \times 7920 \mathrm{AU}$. The spatial sampling is 0 ! $^{\prime} 05$ in right ascencion and 0 '. 10 in declination. In the upper left corner of each panel the corresponding wavelength and atomic/molecular line designation is denoted. In addition, the position of the suspected protostellar source(s) at the disk center is indicated by a cross. In panels c) and e), the knots of the $\mathrm{H}_{2}$ jet are labeled with $\mathrm{A}, \mathrm{B}$, and $\mathrm{C}$.

the kinematics of the innermost part of the disk is not properly traced by the $\mathrm{CO}$ data due to the limited spatial resolution of $\sim 7^{\prime \prime}$ as well as due to the potential confusion by emission from outflowing and infalling material, the millimeter spectroscopy indicates that the disk + envelope system slowly rotates at a velocity of about $0.85 \mathrm{~km} \mathrm{~s}^{-1}$. Dynamical considerations concerning the Keplerian rotation of the outer disk yield a mass of about $15 \mathcal{M}_{\odot}$ for the central protostar. Because the accretion processes are obviously still active (as suggested by the detection of optical emission lines; Chini et al. 2004) and because the gas reservoir of the disk still allows for substantial mass gain, we argue that in the case of the M 17 silhouette disk a massive protostar is on its way to becoming an O-type star.

In this paper, we report and discuss integral field spectroscopic data of the M 17 silhouette disk that were obtained in the $K$-band with VLT + SINFONI. These data allow us to add another important piece to the puzzle of this truly interesting source, and help us to characterize and understand the on-going physical processes.

\section{Observations and data reduction}

The Adaptive Optics (AO) supported near-IR integral field spectrograph SINFONI (Spectrograph for INtegral Field Observations in the Near-Infrared) is among the newest additions of instruments to the ESO Paranal observatory (Eisenhauer et al. 2003aa; Bonnet et al. 2004a; Gillessen et al. 2005). It is mounted on the Cassegrain focus of the VLT Yepun and consists of two main components: a curvature sensing AO module, which is a clone of MACAO (Multiple Application Curvature AO), developed and built by ESO (Bonnet et al. 2003, 2004b), and the near-IR spectrograph SPIFFI (SPectrograph for Infrared Faint Field Imaging), developed and built by the Max-PlanckInstitute for Extraterrestrial Physics (MPE, Eisenhauer et al. 2000, 2003b).

The SINFONI data of the M 17 disk $(\mathrm{RA}=18: 20: 26.3$, Dec $=-16: 12: 10, \mathrm{~J} 2000)$ presented here were taken as part of the SINFONI science verification program "Protostellar Disks and Envelopes" (PI D. Nürnberger; see Gillessen et al. 2005) during the nights August 13th/14th, August 14th/15th, and August 20th/21st, 2004. We primarily used the 100 mas pixel scale, covering a field of view of about $3^{\prime \prime} \times 3^{\prime \prime}$ with 32 pixel $\times 64$ pixel of 100 mas $\times 50$ mas each, together with the $K$-band grating, which comprises the wavelength range from about $1.95 \mu \mathrm{m}$ to $2.45 \mu \mathrm{m}$ at the spectral resolution of about $0.5 \mathrm{~nm}$ (dispersion of $0.25 \mathrm{~nm} /$ pixel). In addition, a few data were taken with the same grating in the 250 mas pixel scale mode (field of view of about $8^{\prime \prime} \times 8^{\prime \prime}$ with 32 pixel $\times 64$ pixel of 250 mas $\times 125$ mas each).

AO curvature sensing was performed on the reference source SAO $161357(\mathrm{RA}=18: 20: 27.64$, Dec $=-16: 12: 01.1, \mathrm{~J} 2000$; $V=9.7, J=6^{\mathrm{m}} \cdot 9, H=6^{\mathrm{m}} \cdot 0, K=5.8, L=5.7, M=5.5$ ), which is located at the distance of $21^{\prime \prime} 3$ towards the east of the science target. For proper sky subtraction, immediately after each observation of the science target we observed a reference sky position, applying the fixed offset $\Delta \alpha=-100^{\prime \prime}$ and $\Delta \delta=-45^{\prime \prime}$. The detector integration times (DITs) were $300 \mathrm{~s}$, both for the on-source (target) and the off-source (sky) position. In total, 13 target-sky pairs of high image quality were obtained on a manually applied spatial jitter pattern. Additionally, 
each night the telluric standard Hip 091130 (RA = 18:35:20.47, Dec $=-22: 05: 27.3$, J2000) was observed with the same strategy (auto-jitter pattern, fixed sky offset) as for the science exposures of the M 17 disk, to correct for telluric features and to flux-calibrate the data. However, both telluric line corrections and flux calibrations should be treated with caution as the observations suffered from sky variability as well as from thin cirrus clouds passing through.

Dark frames, lamp flats, and arcs (taken with SINFONI's internal Neon and Argon lamp for the purpose of wavelength calibration) were obtained through the SINFONI scientific calibration plan. Individually for each target-sky pair, all basic steps of data reduction (flat-fielding, sky subtraction, bad pixel and atmospheric distortion correction) as well as the wavelength calibration were performed with the SINFONI data reduction pipeline. Finally, the merging of the fully reduced and wavelength calibrated data cubes of the M 17 disk was performed within IRAF.

\section{Results and discussion}

To provide a finding chart for the SINFONI data, we use high angular resolution $K_{\mathrm{s}}$-band data (wavelength coverage is $\left.\lambda_{\mathrm{c}}=2.18 \pm 0.175 \mu \mathrm{m}\right)$ previously obtained with VLT + NAOSCONICA. The image displayed in the left panel of Fig. 1 is centered on the suspected protostellar source(s) located at the very center of the silhouette disk. It shows a field of view of roughly $27^{\prime \prime} \times 27^{\prime \prime}$, which corresponds to about $60000 \mathrm{AU} \times 60000 \mathrm{AU}$ at the adopted M 17 distance of $2.2 \mathrm{kpc}$. The pixel scaling of 0.027 allows Nyquist sampled imaging at an angular resolution of less than 0.1 (at $2.2 \mu \mathrm{m}$ the diffraction limit of a $8 \mathrm{~m}$ telescope is 57 mas). The area of the VLT + SINFONI study is also indicated.

On the right side of Fig. 1 (panels a-f), we present the results of the SINFONI science verification study of the M 17 silhouette disk. These individual line maps were extracted from the 100 mas data cube, each covering a field of view of about 4.' $8 \times 3$ ". 6 , i.e., $10560 \mathrm{AU} \times 7920 \mathrm{AU}$. The spatial sampling is $0 .{ }^{\prime} 05$ in right ascencion and $0 . ' 10$ in declination. The bandwidth is $0.5 \mathrm{~nm}$, corresponding to one spectral resolution element, centered on the line peak. In the upper left corner of each panel the corresponding (observed) wavelength and the atomic/molecular line designation are denoted. The position of the suspected central protostellar source(s) - for comparison, see the NACO data on the left side of Fig. 1 - is indicated. We emphasize that continuum emission (as determined from adjacent image planes) has been subtracted.

In Fig. 2 we also provide line maps extracted from the data cube taken with the 250 mas pixel scale. Each of them shows a field of view of about $144^{\prime \prime} 0 \times 188^{\prime \prime} 8$, which corresponds to about $30800 \mathrm{AU} \times 41360 \mathrm{AU}$. The individual panels (a-f) are arranged similar to the corresponding ones of Fig. 1.

\subsection{The $\mathrm{H}_{2}$ jet}

\subsubsection{Morphology}

The images produced from the $\mathrm{H}_{2} v=1-0 S(1)(\lambda=2.1221 \mu \mathrm{m})$ and $\mathrm{H}_{2} v=1-0 S(3)(1.9577 \mu \mathrm{m})$ emission clearly reveal a sequence of $\mathrm{H}_{2}$ knots, apparently tracing a collimated jet that emanates from the suspected protostellar source(s) located at the very center of the disk. Its position angle is about $228^{\circ}$, i.e., almost perpendicular to the orientation of the disk plane (position angle $\sim 148^{\circ}$ ). The small deviation of $10^{\circ}$ from perpendicularity is significant, and may suggest precession of the jet around the rotation axis of the disk. Both in the $\mathrm{H}_{2} v=1-0 S(1)$ and the $\mathrm{H}_{2} v=1-0 S(3)$ lines, as well as in the $\mathrm{H}_{2} v=2-1 S(1)$ $(\lambda=2.2478 \mu \mathrm{m})$ line, a faint emission blob is marginally detected just to the west of the disk center. This emission blob might represent the onset of the $\mathrm{H}_{2}$ jet.

Three knots - marked A, B, C in panels c and e of Fig. 1 - of $\mathrm{H}_{2}$ emission are detected at separations of about $0.0^{\prime} 72,1^{\prime \prime} .17$, and 1.' 50 (corresponding to about $1580 \mathrm{AU}, 2570 \mathrm{AU}$, and $3300 \mathrm{AU}$, respectively) from the disk center. However, as obvious from the 250 mas data presented in Fig. 2 (panels $\mathrm{c}$ and e), faint $\mathrm{H}_{2}$ can be found at distances up to 3.' 6 (7920 AU) from the disk center. We note that the potential head of the jet approximately coincides (by chance) with rather strong, point-like $\mathrm{H}_{2}$ emission, indicated in Fig. 2 (panels a, c, and e). This point-like emission almost disappears after appropriate continuum subtraction (not shown in Fig. 2) and has an unambiguous counterpart in the NACO broad-band $K_{\mathrm{s}}$ image (Fig. 1, left panel). Therefore, we believe that it mostly consists of continuum emission that arises from a foreground/background star unrelated to the $\mathrm{H}_{2}$ jet.

No counter jet has been found on the other side of the circumstellar disk. This is not surprising as any potential counter jet is ejected straight in the direction of the neighboring mainsequence OB stars, which is expected to be mostly void of interstellar material as it is exposed to strong stellar winds and ionizing photons. Any counter jet might possibly not find (dense) enough interstellar material to disclose its identity by shockexcited $\mathrm{H}_{2}$ emission. In addition, ejected material by itself is eventually affected (deflected and squeezed back to the disk surface) by the incoming wind and ionization front. In fact, this picture might be corroborated by optical and near-IR imaging data (see Fig. 4 of Chini et al. 2004), showing a bipolar reflection nebula that (integrated over all wavelengths) appears much more compact on the OB stars facing side than on the other side of the disk.

Because ejection of material through a jet/outflow is always linked to accretion of gas and dust either onto the circumstellar disk or onto the central (protostellar) source(s), in the case of the M 17 disk the presence of the $\mathrm{H}_{2}$ jet provides indirect but unquestionable evidence for ongoing accretion processes. This conclusion is in agreement with the findings from optical spectra of the bipolar reflection nebula (Fig. 5 of Chini et al. 2004), which exhibits several emission lines - e.g., $\mathrm{H} \alpha$, the Ca II $8498 \AA, 8542 \AA$, and $8662 \AA$ triplet and the He I $6678 \AA$ line indicative of the accretion of material from the inner disk onto the central star.

\subsubsection{Physical parameters}

In Table 1 we list the measured $\mathrm{H}_{2}$ line intensities for each of the three knots. Our data set yields detections of the 1-0 S(1) and $1-0 S(3) \mathrm{H}_{2}$ lines towards all three knots $\mathrm{A}, \mathrm{B}$, and $\mathrm{C}$. The $1-0 S(1) / 1-0 S(3)$ intensity ratios are around unity and below, which might suggest that shocks are the most likely excitation mechanism. However, the upper limits derived for the 2-1S(1) line, which are about $60-90 \%$ of the $1-0 S(1)$ line instensities, are not exceedingly meaningful to constrain rotational excitation temperatures and the excitation mechanism. From the literature it is well known that fluxes in the 2-1S(1) line are typically 10 $20 \%$ compared to those in the 1-0 S (1) line for shock-excited molecular gas (e.g., Caratti o Garatti et al. 2006; Gredel 2006). In contrast, in case of excitation by UV fluorescence and subsequent decay through a cascade of ro-vibrational transitions, the same flux ratio is expected to reach values of $50 \%$ and beyond 

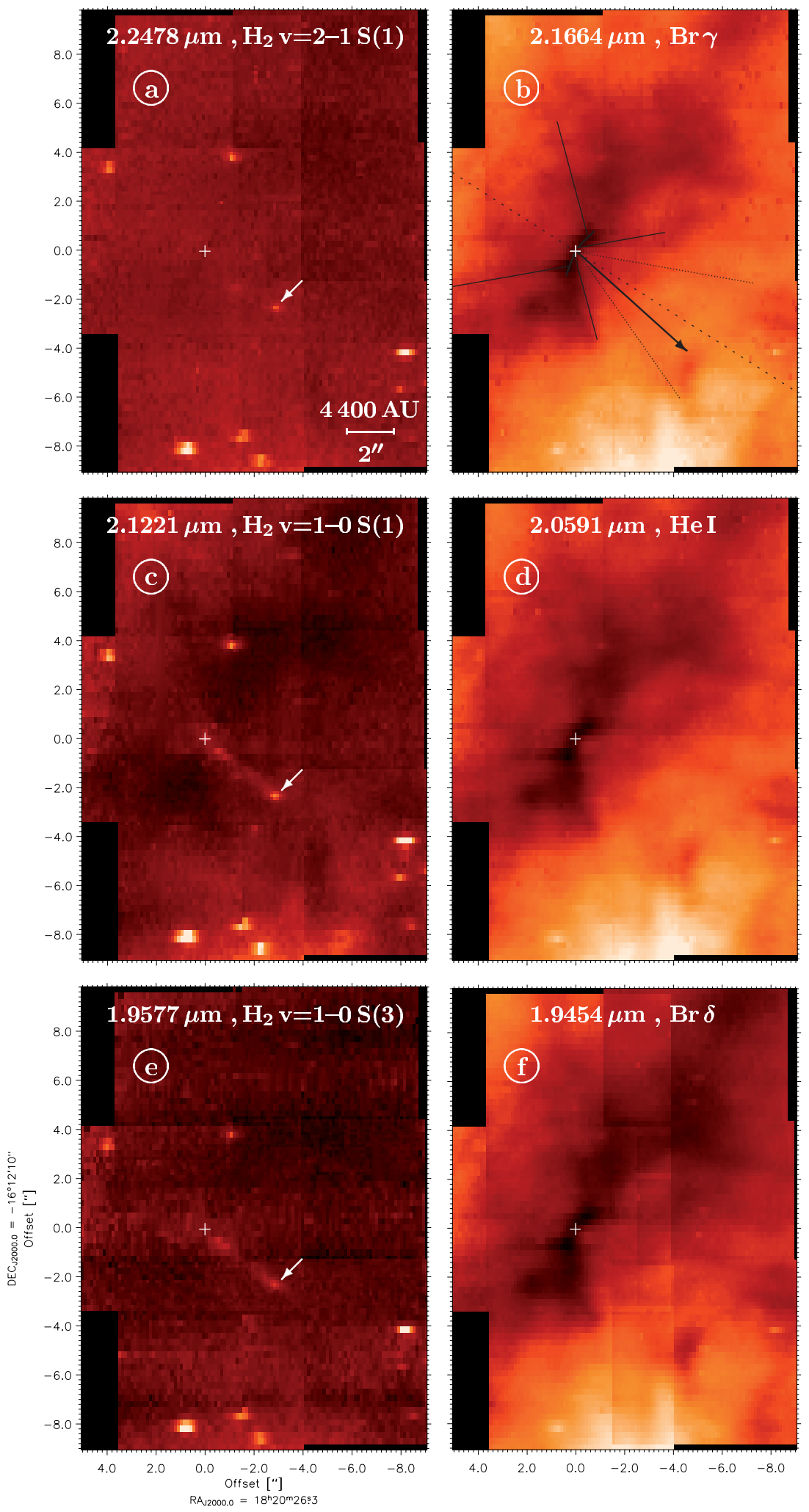

Fig. 2. Selected line maps extracted from the SINFONI data cube taken with the 250 mas pixel scale. The spatial sampling is 0 '. $^{\prime} 125$ in right ascencion and 0 '.25 in declination. Each image comprises a field of view of about $14{ }^{\prime \prime} 0 \times 18^{\prime \prime} .8$, corresponding to about $30800 \mathrm{AU} \times 41360 \mathrm{AU}$ at the adopted M 17 distance of $2.2 \mathrm{kpc}$. The individual panels $\mathbf{a})-\mathbf{f})$ are arranged similarly to the corresponding ones of Fig. 1. At the top of each panel the corresponding wavelength and atomic/molecular line designation is denoted. The position of the suspected protostellar source(s) at the disk center is indicated by a cross. In panels a), c), and e), an arrow indicates a foreground/background star, which is by chance located in the direction of the $\mathrm{H}_{2}$ jet. In panel b) we also overplot a sketch that is meant to help the reader with the orientation of the M 17 silhouette disk (thick straight lines) and of its rotation axis (thin dashed line). The walls of the outflow cavities are outlined by thin straight lines; in particular, one should notice the wider opening angle of the outflow cavity on the north-east side of the disk, facing the nearby mainsequence $\mathrm{O}$ type stars. The orientation of the $\mathrm{H}_{2}$ jet is marked by the thick arrow pointing towards the southwest of the disk; the deviation from the rotation axis of the disk is obvious and most likely explained by precession of the jet. The proposed precession cone is outlined by thin dotted lines. (e.g., Black \& Dalgarno 1976; Black \& van Dishoeck 1987; Draine \& Bertoldi 1996). Therefore, we cannot rule out excitation by fluorescence.

Nevertheless, without having deeper spectra at hand to detect the $\mathrm{H}_{2} 2-1 S$ (1) line, we will first take the 2-1 $S$ (1) upper limits at face value and use them for the derivation of physical parameters. Afterwards, we will probe the validity of this assumption and of the obtained results by explicitely replacing the upper limits for the 2-1S(1) line by fluxes corresponding to 10 $20 \%$ (shock-excited $\mathrm{H}_{2}$; case A) and $50 \%$ (fluorescently excited $\mathrm{H}_{2}$; case B) of those measured in the 1-0 S (1) line for each knot.

\section{Parameters as derived from the SINFONI data}

The reddening is generally determined from the ratio of two ro-vibrational lines that share the same upper level. For such 
Table 1. Physical parameters derived from the observed $\mathrm{H}_{2}$ line emission.

\begin{tabular}{|c|c|c|c|c|c|c|c|c|c|}
\hline \multirow[t]{2}{*}{ Parameter } & \multicolumn{3}{|c|}{$\overline{\mathrm{Knot} A}$} & \multicolumn{3}{|c|}{ Knot B } & \multicolumn{3}{|c|}{ Knot C } \\
\hline & $1-0 S(3)$ & $1-0 S(1)$ & $2-1 S(1)$ & $1-0 S(3)$ & $1-0 S(1)$ & $2-1 S(1)$ & $1-0 S(3)$ & $1-0 S(1)$ & $2-1 S(1)$ \\
\hline$d$ arcsec] $^{[a]}$ & & 0.72 & & & 1.17 & & & 1.50 & \\
\hline$I\left(\mathrm{H}_{2}\right)\left[\frac{\mathrm{W}}{\mathrm{m}^{2} \operatorname{arcsec}^{2}}\right]$ & $1.4 \times 10^{-18}$ & $1.4 \times 10^{-18}$ & $<8.4 \times 10^{-19}$ & $1.7 \times 10^{-18}$ & $1.2 \times 10^{-18}$ & $<8.4 \times 10^{-19}$ & $2.0 \times 10^{-18}$ & $9.3 \times 10^{-19}$ & $<8.4 \times 10^{-19}$ \\
\hline$S / N[\sigma]$ & $\sim 3$ & $\sim 5$ & $<3$ & $\sim 3$ & $\sim 4$ & $<3$ & $\sim 4$ & $\sim 3$ & $<3$ \\
\hline$A_{K}[\mathrm{mag}]^{[b]}$ & & 14.6 & & & 6.7 & & & 0.4 & \\
\hline$F\left(\mathrm{H}_{2}\right)\left[\frac{\mathrm{W}}{\mathrm{m}^{2}}\right]^{[b],[c]}$ & $5.8 \times 10^{-11}$ & $5.9 \times 10^{-11}$ & $<3.5 \times 10^{-11}$ & $5.1 \times 10^{-14}$ & $3.4 \times 10^{-14}$ & $<2.5 \times 10^{-14}$ & $1.9 \times 10^{-16}$ & $8.8 \times 10^{-17}$ & $<7.9 \times 10^{-17}$ \\
\hline $\mathcal{L}\left(\mathrm{H}_{2}\right)\left[\mathcal{L}_{\odot}\right]^{[b],[c]}$ & 8.8 & 8.9 & 5.3 & $7.7 \times 10^{-3}$ & $5.2 \times 10^{-3}$ & $3.8 \times 10^{-3}$ & $2.8 \times 10^{-5}$ & $1.3 \times 10^{-5}$ & $<1.2 \times 10^{-5}$ \\
\hline
\end{tabular}

Notes: ${ }^{a}$ Measured from the disk center; ${ }^{b}$ highly uncertain, as based on upper limits for the $\mathrm{H}_{2} v=2-1 \mathrm{~S}(1)$ line fluxes (see detailed discussion in the text); ${ }^{c}$ extinction corrected.

line pairs, one can use formulae 1 and 2 of O'Connell et al. (2005) to estimate the line-of-sight extinction towards each $\mathrm{H}_{2}$ knot. Using the $1-0 S(1)$ fluxes together with the $2-1 S(1)$ upper limits, and assuming a $K$-band spectral index of -0.72 (as derived from the continuum emission underlying the line emission), we obtain $A_{\mathrm{K}}$ estimates of $14 . \mathrm{m}, 6,6.7$, and 0.4 for knot $\mathrm{A}, \mathrm{B}$, and $\mathrm{C}$, respectively. Applying transformations given by Rieke \& Lebofsky (1985), these values convert to visual extinctions of $A_{\mathrm{V}} \sim 146^{\mathrm{m}}, 67^{\mathrm{m}}$, and $4^{\mathrm{m}}$. The decrease of extinction with increasing distance from the midplane of the circumstellar disk appears consistent with the (expected) decrease of the amount of intrinsic disk/envelope material for the three lines-of-sight. The $A_{\mathrm{V}}$ value of knot $\mathrm{C}$ is in good agreement with the minimum extinction $\left(A_{\mathrm{V}} \gtrsim 3.4\right)$ derived from near- and mid-IR photometry of young (class I) high-mass sources located in the neighborhood of the silhouette disk (Nielbock et al. 2001). This minimum extinction is convincingly explained by foreground extinction, considering a distance of $2.2 \mathrm{kpc}$ for M 17 and (by rule-of-thumb) an average visual extinction of 2 mag per kpc for the Galactic plane.

With $A_{K}$ values at hand, we can derive extinction-corrected $\mathrm{H}_{2}$ fluxes and $\mathrm{H}_{2}$ luminosities as summarized in Table 1. At this point, we refrain from a major discussion on which fraction of the total $\mathrm{H}_{2}$ flux (or luminosity) is actually emitted in the observed lines. For $\mathrm{H}_{2}$ jets associated with low-mass (proto-) stars, this fraction is typically of the order of $10 \%$ in the case of the $1-0 S(1)$ and 1-0 S (3) lines, respectively. Therefore, the total $\mathrm{H}_{2}$ luminosity of our three jet knots might amount to about $88 \mathcal{L}_{\odot}$. Moreover, assuming that the total $\mathrm{H}_{2}$ luminosity might contribute only $1 \%$ to the bolometric luminosity of the knots (e.g., Smith 1995), the latter may be as high as $8.8 \times 10^{3} \mathcal{L}_{\odot}$. Based on the rotational diagram and assuming local thermodynamical equilibrium (LTE) for the observed $\mathrm{H}_{2}$ lines, we derive excitation temperatures of the order $T_{e x}=8600-12700 \mathrm{~K}$, column densities in the range $\mathcal{N}=8.1 \times 10^{24}-1.9 \times 10^{19} \mathrm{~m}^{-2}$, as well as mass estimates between $\mathcal{M}=0.3 \mathcal{M}_{\odot}($ knot A) and $\mathcal{M}=7 \times 10^{-7} \mathcal{M}_{\odot}($ knot $\mathrm{C})$. We emphasize that these excitation temperatures, column densities, and mass estimates must be considered highly uncertain (by orders of magnitude; see also the case study further below), as they are based on upper limits for the 2-1 $S$ (1) line fluxes.

Because the knot luminosity itself represents the rate of energy that is fed into the jet by the driving source, we can estimate the mass outflow rate $\dot{\mathcal{M}}_{\text {out }}=2 \times \mathcal{L} \times v_{\text {jet }}^{-2}$ and - as outflow and accretion processes are closely linked to each other the (proto-) stellar mass accretion rate $\dot{\mathcal{M}}_{\text {acc }}=\epsilon^{-1} \dot{\mathcal{M}}_{\text {out }}$. If we assume the value of $\epsilon$ to be of the order of 0.3 (like in diskwind models; see, e.g., the discussion in Stanke 2000, and references therein) and the typical jet velocity to be $100 \mathrm{~km} \mathrm{~s}^{-1}$, we obtain - based on the pure $\mathrm{H}_{2}$ luminosities - a mass outflow rate and mass accretion rate of $\dot{\mathcal{M}}_{\text {out }} \sim 2.8 \times 10^{-5} \mathcal{M}_{\odot} \mathrm{yr}^{-1}$ and $\dot{\mathcal{M}}_{\text {acc }} \sim 9.3 \times 10^{-5} \mathcal{M}_{\odot} \mathrm{yr}^{-1}$, respectively. In comparison to typical mass outflow/accretion rates observed for low-mass protostars $\left(10^{-6}-10^{-7} \mathcal{M}_{\odot} \mathrm{yr}^{-1}\right)$, these values appear rather high. However, we note that they may represent lower limits only as no conversion from $\mathrm{H}_{2}$ to bolometric luminosities has been applied. In fact, accretion rates in excess of $10^{-5} \mathcal{M}_{\odot} \mathrm{yr}^{-1}$ are expected to be observed for intermediate-mass and high-mass (proto-) stars (e.g., Norberg \& Maeder 2000; and McKee \& Tan 2002, 2003).

\section{Reliability/uncertainty of the derived parameters}

Finally, some words on the reliability of the estimated physical parameters of the jet. As is obvious from the $S / N$ values provided in Table 1, the measured line intensities are quite weak. They should be considered uncertain by a factor of 2 due to imperfectness of the data reduction and data calibration (see Sect. 2). This uncertainty basically propagates in the subsequent derivation of all other parameters. The reliability of the estimated mass outflow/accretion rate mostly depends on the underlying assumptions as discussed above. E.g., when keeping all other parameters unchanged, a variation of the $K$-band spectral index by \pm 0.1 (i.e., from -0.82 to -0.62 ) would yield (pure $\mathrm{H}_{2}$ ) mass outflow/accretion rates in the range $\dot{\mathcal{M}}_{\text {out }} \sim 6 \times 10^{-6}$ $2 \times 10^{-4} \mathcal{M}_{\odot} \mathrm{yr}^{-1}$ and $\dot{\mathcal{M}}_{\mathrm{acc}} \sim 2 \times 10^{-5}-7 \times 10^{-4} \mathcal{M}_{\odot} \mathrm{yr}^{-1}$, respectively.

Much larger uncertainties are introduced by the use of upper limits for 2-1 $S$ (1) line fluxes. While keeping all other parameters unchanged, what happens if we replace the 2-1 $S$ (1) upper limits by fluxes corresponding to either 10-20\% (shock-excited $\mathrm{H}_{2}$; case A) or $50 \%$ (fluorescently excited $\mathrm{H}_{2}$; case B) of those measured in the 1-0S(1) line for each knot?

In case $\mathrm{A}$, as derived from the rotation diagram, the excitation temperatures drop to roughly $2500-3000 \mathrm{~K}$. However, the line-of-sight extinction towards all three $\mathrm{H}_{2}$ knots becomes extremely high: $A_{\mathrm{K}} \sim 40^{\mathrm{m}}$ or $A_{\mathrm{V}} \sim 400^{\mathrm{m}}$. As a consequence, dereddened $\mathrm{H}_{2}$ line fluxes and luminosities reach very high values: $F\left(\mathrm{H}_{2}\right) \sim 10^{-5}-10^{-4} \mathrm{~W} \mathrm{~m}^{-2}$ and $\mathcal{L}\left(\mathrm{H}_{2}\right) \sim 10^{10}-10^{11} \mathcal{L}_{\odot}$. Mass outflow rates and mass accretion rates go beyond the ceiling.

In case $\mathrm{B}$, the line-of-sight extinction towards all three $\mathrm{H}_{2}$ knots reaches values of $A_{\mathrm{K}} \sim 20^{\mathrm{m}}$ or $A_{\mathrm{V}} \sim 200^{\mathrm{m}}$. The dereddened $\mathrm{H}_{2}$ line fluxes and luminosities are on the order of $F\left(\mathrm{H}_{2}\right) \sim 10^{-12}-10^{-11} \mathrm{~W} \mathrm{~m}^{-2}$ and $\mathcal{L}\left(\mathrm{H}_{2}\right) \sim 10^{3}-10^{4} \mathcal{L}_{\odot}$, respectively. $\mathrm{H}_{2}$ mass outflow rates and mass accretion rates are still rather high, i.e., about $\dot{\mathcal{M}}_{\text {out }} \sim 4 \times 10^{-2} \mathcal{M}_{\odot} \mathrm{yr}^{-1}$ and $\dot{\mathcal{M}}_{\text {acc }} \sim$ $1 \times 10^{-1} \mathcal{M}_{\odot} \mathrm{yr}^{-1}$. As derived from the rotation diagram, the excitation temperatures would drop to about 4700-6000 K. 
On the one hand, these considerations demonstrate that the usage of 2-1 $S$ (1) upper limits leads to valid conclusions, i.e., reasonable (and strict) lower limits of mass outflow rates and mass accretion rates. On the other hand, the large uncertainties in the line fluxes very severely limit the determination of excitation temperatures. In any case, inferred excitation temperatures of $8600-12700 \mathrm{~K}$ are too high if the emission were to arise from shock-excited gas. While recent observations show that a small fraction of shock-heated gas may be at temperatures above $5000 \mathrm{~K}$, the bulk emission arises from gas at temperatures of typically $2000 \mathrm{~K}$ (Gredel 2006; Caratti o Garatti et al. 2006). However, our significant overestimation of excitation temperatures might (in part) also be caused by the relatively small range in excitation energies $-8365 \mathrm{~K}, 6956 \mathrm{~K}$, and $12550 \mathrm{~K}$ for $1-$ $0 S(3), 1-0 S(1)$, and 2-1 S(1), respectively - of the corresponding upper ro-vibrational levels of the studied $\mathrm{H}_{2}$ lines.

\subsection{The silhouette disk}

Through the direct comparison of the NACO and the SINFONI data, it is evident that the picture derived from the $\mathrm{Br} \gamma$ $(\lambda=2.1664 \mu \mathrm{m})$, He I $(2.0591 \mu)$, and $\operatorname{Br} \delta(2.1664 \mu \mathrm{m})$ line emission closely resembles that obtained from the broadband $K_{\mathrm{s}}$ emission. The silhouette disk is seen almost edge-on and shows a flared character. Even more than in the continuum, the silhouette of the disk is seen in absorption against the extended $\mathrm{Br} \gamma, \mathrm{He} \mathrm{I}$, and $\operatorname{Br} \delta$ background emission. Given the relatively small field of view of the SINFONI data (both 100 mas and 250 mas data sets) in comparison to that of the NACO data, we lack a suitable reference position to reliably measure the (unabsorbed) background emission arising from the M $17 \mathrm{H}$ II region. Therefore, we do not attempt to derive a dust extinction map from the SINFONI data. Instead, we refer to the recent work of Steinacker et al. (2006), who have done a detailed modeling of the NACO data of the M 17 disk.

From both the NACO and the SINFONI data sets, we derive a diameter of about $4000 \mathrm{AU}$ (1'.8) for the innermost densest part of the disk. Furthermore, in the SINFONI data it also appears shaped wing-like which is most likely caused by slight inclination (about $10^{\circ}$ ) of the disk plane against the line-of-sight. A similar scenario has been discussed recently by Smith et al. (2005 in particular, see their Figs. 1g and 2) for the newly discovered giant silhouette disk d216-0939 located north of the Orion Nebula and northwest of M 43. The plane of this disk is obviously inclined against the plane of the sky by roughly $83^{\circ}$, resulting in an overall morphology (wing-like, flared, etc.) very similar to the M 17 silhouette disk.

An alternative explanation for the wing-like shape of the M 17 silhouette disk might be given by the external pressure which is exerted on the disk surface by both ionizing photons and strong winds originating from the nearby main-sequence OB stars of the M 17 cluster. A similar wing-like appearance can be reproduced by numerical model calculations of externally illuminated and photoevaporated protostellar disks (Richling \& Yorke 1998, e.g., see their Figs. 6 and 9). In the same sense, the outflow cavity towards the north-east (i.e., on the OB stars facing side of the disk) apparently has a much wider opening angle than the outflow cavity towards the south-west (i.e., on the side of the disk opposite the OB stars). This points towards a stronger interaction of outflowing material with the incoming wind/ionization front on the disk side that is directly exposed to the nearby massive main-sequence stars. The counter outflow might be less affected by these interactions as it is largely shielded by the disk.

Acknowledgements. Sylvie Cabrit and Michael Smith are thanked for helpful discussions on the interpretation of $\mathrm{H}_{2}$ line emission. In addition, insightful comments of the anonymous referee are acknowledged.

\section{References}

Beech, M. \& Mitalas, R. 1994, ApJS, 95, 517

Behrend, R., \& Maeder, A. 2001, A\&A, 373, 190

Bernasconi, P. A., \& Maeder, A. 1996, A\&A, 307, 829

Beuther, H., \& Schilke, P. 2004, Science, 303, 1167

Black, J. H., \& Dalgarno, A. 1976, ApJ, 203, 132

Black, J. H., \& van Dishoeck, E. F. 1987, ApJ, 322, 412

Bonnell, I. A. 2002, in Hot Star Workshop III: The Earliest Stages of Massive Star Birth, ed. P. A., Crowther, ASP Conf. Proc., 267, 193

Bonnell, I. A., \& Bate, M. R. 2002, MNRAS, 336, 659

Bonnell, I. A., Bate, M. R., \& Zinnecker, H. 1998, MNRAS, 298, 93

Bonnet, H., Ströbele, S., Bianca-Marchet, F., et al. 2003, SPIE, 4839, 329

Bonnet, H., Abuter, R., Baker, A., et al. 2004a, ESO Messenger, 117, 17

Bonnet, H., Conzelmann, R., Delabre, B., et al. 2004b, SPIE, 5490, 130

Caratti o Garatti, A., Giannini, T., Nisini, B., \& Lorenzetti, D. 2006, A\&A, 449, 1077

Chini, R., Hoffmeister, V., Kimeswenger, S., et al. 2004, Nature, 429, 155

Draine, B. T., \& Bertoldi, F. 1996, ApJ, 468, 269

Eisenhauer, F., Tecza, M., Mengel, S., et al. 2000, SPIE, 4008, 289

Eisenhauer, F., Bonnet, H., Abuter, R., et al. 2003a, SPIE, 4841, 1548

Eisenhauer, F., Tecza, M., Thatte, N., et al. 2003b, ESO Messenger, 113, 17

Gillessen, S., Davies, R., Kissler-Patig, et al. 2005, ESO Messenger, 120, 26

Gredel, R. 2006, A\&A, 457, 157

McKee, C. F., \& Tan, J. C. 2002, Nature, 416, 59

McKee, C. F., \& Tan, J. C. 2003, ApJ, 585, 850

Nielbock, M., Chini, R., Jütte, M., \& Manthey, E. 2001, A\&A, 377, 273

Norberg, P., \& Maeder, A. 2000, A\&A, 359, 1025

Nürnberger, D. E. A. 2003, A\&A, 404, 255

Nürnberger, D. E. A. 2004, Ph.D. Thesis, Julius-Maximilians-Universität Würzburg

Nürnberger, D. E. A., Chini, R., \& Hoffmeister, V. H. 2005, in The Nature and Evolution of Disks around Hot Star, ed. R., Ignace, K., Gayley, ASP Conf. Ser., 337, 279

O'Connell, B., Smith, M. D., Froebrich, D., Davis, C. J., \& Eislöffel, J. 2005, A\&A, 431, 223

Richling, S., \& Yorke, H. W. 1998, A\&A, 340, 508

Rieke, G. H., \& Lebofsky, M. J. 1985, ApJ, 288, 618

Smith, M. D. 1995, A\&A, 296, 789

Smith, N., Bally, J., Licht, D., \& Walawender, J. 2005, AJ, 129, 382

Stanke, T. 2000, Ph.D. Thesis, Universität Potsdam

Steinacker, J., Chini, R., Nielbock, M., et al. 2006, A\&A, 456, 1013

Yorke, H. W. 1986, ARA\&A, 24, 49

Yorke, H. W., \& Sonnhalter, C. 2002, ApJ, 569, 846 